\title{
Cost-efficient Traffic-aware Data Collection Protocol in VANET
}

\author{
Zongjian He, Daqiang Zhang \\ School of Software Engineering, Tongji University, Shanghai, China, 201804
}

\begin{abstract}
Data from running vehicles are invaluable to numerous ITS and urban computing applications. This paper studies the issue of collecting data from multiple vehicles to a roadside base station via vehicle-to-vehicle and vehicle-toinfrastructure communications. Existing data collection approaches in VANET have mainly focused on the network problems, such as packet loss, network clustering or data aggregation, but the impact of real-time traffic condition is barely considered. In this paper, we investigate the data collection problem in VANETs under rapid evolving traffic conditions. Our approach can adaptively choose to carry or forward the data packet, based on current traffic information. The objective is to minimize the network communication overhead while satisfying the data collection time constraint. We formulate the data collection problem as a scheduling optimization problem and prove it is NP-complete. An optimal dynamic programming solution and a genetic algorithm based heuristic solution are developed to solve the problem under different application scenarios. Extensive evaluations validate that our proposed solution outperforms some existing ones in terms of effectiveness and efficiency.
\end{abstract}

Keywords: Data collection, VANET, traffic information, dynamic programming, genetic algorithm.

\section{Introduction}

Over the past decades, we have witnessed the development of automotive electronics [1] and IoTs [2]. Nowadays, modern vehicles have already equipped with dozens or even hundreds of sensors onboard, like oxygen sensor, speedometer, temperature sensor and etc [3]. Undoubtedly, the sensory data can contribute useful information to many emerging intelligent transportation system and urban computing applications [4] [5], such as traffic congestion detection [6], vehicle emission and —environmental pollution monitoring [7]. Currently, many commercialized systems like WAZE (http://www.waze.com) utilize cellular network (UMTS/LTE) to collest data from vehicles. However, the cellular

Email address: \{hezongjian, dqzhang\}@tongji.edu.cn (Zongjian He, Daqiang Zhang) 


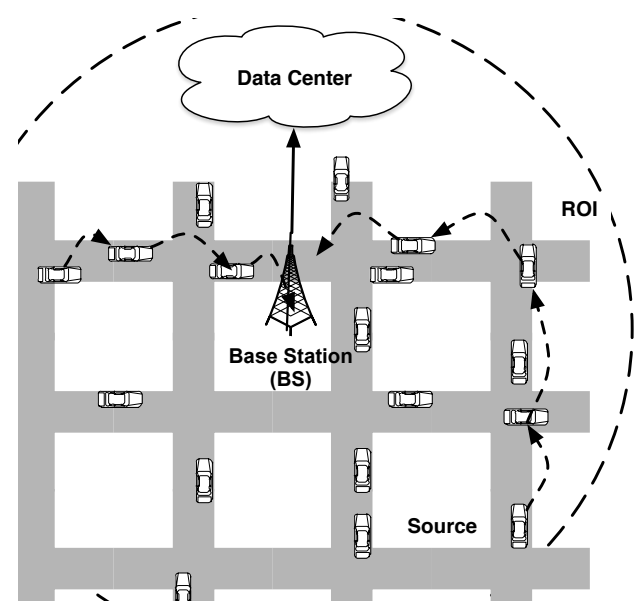

Figure 1: The urban data collection scenario

communication brings remarkably expensive communication cost and hinders the popularization of these systems.

With the advances of communication technology, vehicular ad-hoc network (VANET) becomes a prominent network paradigm. Using VANET, vehicles can not only communicate with nearby neighbors (vehicle to vehicle, $\mathrm{V} 2 \mathrm{~V}$ ), but also exchange information with roadside infrastructures (vehicle to infrastructure, V2I). With the help of VANET, collecting sensory data from vehicles without using the expensive cellular communication becomes realistic: data can first be collected to roadside base stations using V2V/V2I communication. Then, the base stations can directly pass the collected data to data center with backbone networks.

This paper studies the problem of data collection using VANET in urban area. The application scenario is illustrated in Fig. 1. A fixed base station (BS) is deployed at the roadside as sink node. The BS is responsible for collecting data from surrounding vehicles within a specific region of interest (ROI). A complete data collection process is composed of two phases: First, the BS sends a query to its nearby vehicles within the ROI in multi-hop. After a vehicle with requested data receives the query, it will send the data back to BS also via multi-hop relay.

This data collection scenario raises a number of challenges. First, for applications with real-time requirement, the value of the data decreases rapidly with time. Therefore, the timeliness of data is crucial and data must be collected within a tolerable delay. Next, the communication in VANETs is highly affected by traffic condition [8, 9] which changes over time. Hence, the data collection solution must be adaptive to the current traffic condition for performance consideration. Last, in urban scenario, the number of vehicles involved could be potentially large. Moreover, applications may require not only primitive sensor reading but also multimedia data like video streaming or high definition photos. 
Therefore, the amount of data to be transferred could be large and the network communication overhead must be considered by the data collection protocol.

To handle these challenges, active researches have been conducted in this area. Researchers have proposed a number of VANET based protocols, such as Mobeyes [10, CGP [11] and DB-VDG [12. These pioneer works have brought new insights into the VANET based data collections. However, none of them have considered the impact of real-time traffic information in the protocol design. Differently, we propose a Real-time traffic Information aware Data collEction solution, or RIDE. In RIDE, data from surrounding vehicles must be collected to BS within predefine delay constraint. Considering the diversity of traffic condition, the next hop node in VANET is not always available. Therefore, data should be carried by vehicles in this case. On the other hand, if the time constraint is not tight, asking vehicles driving towards the base station to carry the packets can reduce the network communication overhead. In our approach, RIDE can schedule data transmission adaptively according to the real-time traffic information. Specifically, the data can either be forwarded by vehicular network toward BS, or be carried by certain vehicles driving toward BS. In addition, RIDE can forward data only when necessary to minimize the network communication overhead.

To the best of our knowledge, we are the first to design a real-time traffic adaptive protocol for VANET based data collection. The contributions of this paper can be summarized as follows:

- We formulate the RIDE problem rigorously as an optimization problem. Then, we prove the problem belongs to NP-complete.

- We propose two solutions for this problem under different application scenarios: the dynamic programming based solution for small-scaled data collection and the genetic algorithm based solution for large-scaled data collection.

- Empirical evaluations are conducted using real traffic trace. The evaluation results show that RIDE outperforms some existing solutions in terms of effectiveness and efficiency.

The rest of this paper is organized as follows. Related works are summarized in Section 2. In Section 3 , the RIDE problem is formulated and NP-completeness is proved. Section 4 proposes two solutions for RIDE, one is dynamic programming based and the other is genetic algorithm based. After that, the design of network protocol is introduced in Section 5. In Section 6, the solution is evaluated and the results are explained. Finally, Section 8 concludes this paper.

\section{Related Work}

\subsection{Data Collection in VANETs}

All data collection solutions in VANETs have to consider some common functional requirements. We can use a $3 \mathrm{D}$ functional framework which is depicted 
in Fig. 2 to categorize these requirements. Node mobility refers to whether the sensor or sink nodes are mobile. Data initiator indicates how to initiate the data collection, and Metric of interest means what is the primary concern of the solution. We will use this framework to categorize the related works.

Mobeyes [10] is a disruption tolerance and scalability vehicular sensing platform designed for urban data collection. The Mobeyess on-demand data harvest mode deals with similar problem with us except collection ratio is concerned (MS- PL-CR). Unlike Mobeyes which tries to construct index for nearby vehicles, our solution is structure free and do not have additional index maintenance cost.

CGP 11 is a geographical cluster based data collection protocol for VANETs. Data within clusters are firstly collected to the cluster head and then transferred to the sink. CGP is also similar except data are pushed to sink (MS-PS-CC). The data collection process is simplified with only one-way communication. Moreover, CGP is designed for a single road and maybe not be directly applicable for large region due to the cluster management overhead.

SMITE [13 is a stochastic, structure free data collection protocol (MM-PL). Both sensors and sink are mobile. It randomly selects some intermediate collector to collect data from nearby and then transfer data to sink using geographical routing. SMITE tries to optimize LT, CR and CC on a best- effort basis, but no guaranteed service is provided.

PBS [14 is a structured data harvest algorithm that can be classified as SM-PL-CR with only one mobile sink. It uses KD-tree to partition nodes into separate clusters. Then data can be collected within each cluster. The advantage of KD-tree is that the overhead of update is small thus the complexity of the algorithm can be bounded.

DB-VDG [12] is a delay-bounded vehicular data collection protocol. DBVDG deals with the same problem with us (MS-PL-CC) and can be treated as state-of-the-art solution. In DB-VDG, vehicles try to carry data as long as possible to save communication cost. Differently from DB-VDG, our solution consider the real-time traffic information, the decision whether to carry or to forward data is not only determined by time boundary but also by traffic condition.

We categorize our study as MS-PL-CC, which means in our solution vehicles are mobile but the sink are static (MS), data collection request is initiated by sink (PL) and we want to optimize the communication cost (CC).

In summary, data collection is a well investigated topic in wireless networks. However, if we categorize existing works using the 3D functional framework in fine granularity, few works fall into the same category (MS-PL-CC) with us. The closest work is DB-VDG [12, which will be compared in evaluation.

\subsection{Routing in Delay Tolerant Networks}

The RIDE problem shares some similarity with conventional delay tolerant networks (DTN). Therefore, we also review some protocol designs in DTN. 


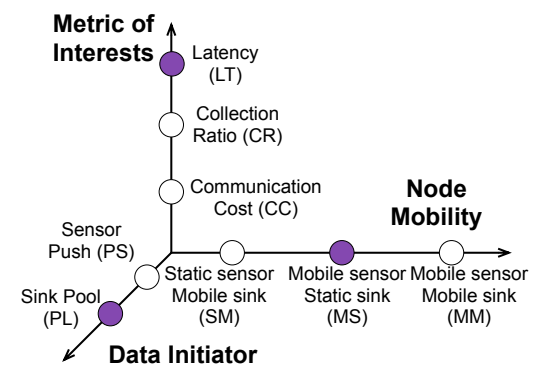

Figure 2: 3D functional framework for data collection in VANETs

MaxProp 15] is a DTN routing protocol aiming at increasing delivery rate and reduce latency. It works in a storage and bandwidth constrained environment. It utilizes historical data to calculate packet priority for sending or dropping. RAPID [16 treats DTN routing as a resource allocation problem and transform the routing metric int o per-packet utilities. Thus it can be used to maximizes the performance of a specific routing metric. DEAR [17] proposed a distance aware DTN routing based on real traffic trace to increase the delivery ratio. However, the dynamicity of the traffic information is not considered.

\section{Problem Statement}

\subsection{Preliminaries}

In this research, we assume vehicles are able to determine their locations through GPS and digital map, and can establish vehicular network connections using standard wireless communication protocols like IEEE 802.11p. We denote the mean transmission range of vehicular network as $r$ and the mean one-hop transmission latency as $t$. Notice that mean transmission range is different from the maximum communication distance. We set $r$ to be half of the maximum communication distance according to probability expectation.

Definition 1. (Real-time Traffic Map) The real-time traffic map contains static road network and dynamic real-time traffic information. The road network can be defined as a planar graph $R T M=(N, E)$, where $E$ is the set of all road segments and $N$ is the set of all road intersections. With real-time traffic information, a road segment $s$ can be defined as a 3-tuple $\{l, v, d\}$, where $l(s)$ is the length of the road segment. $v(s)$ and $d(s)$ are the mean vehicle speed and vehicle number on the road segment, respectively. The beginning and ending point of $s$ can be denoted as $b(s)$ and $e(s)$.

Parameters like road segment length and vehicle density are essential to the accuracy of the system. Therefore, it is important to clarify the ways to obtain them. Generally, the length $l(s)$ of a road segment $s$ can be obtained via the digital maps installed on vehicle's navigation system or online map service 
Table 1: Summary of Notations

\begin{tabular}{c|c}
\hline Symbols & Definition \\
\hline$r$ & The average network transmission range for one hop. \\
$R T M$ & The average transmission latency for one hop. \\
$s_{i}$ & The real-time traffic map with $(N, E)$. \\
$l(s), v(s), d(s)$ & A road segment $i$ in $R T M$ with 3 -tuple $(l, v, d)$. \\
$b(s), e(s)$ & Length, speed or vehicle number of the road segment. \\
$o$ & Starting and ending intersection of a road segment. \\
$c$ & The location of base station infrastructure. $o \in N$. \\
$\mathcal{P}$ & The location of collected vehicle. $c \in N$. \\
$\left(s_{i}, x_{i}\right)$ & A complete schedule policy in $R T M$. \\
$F$ & Scheduling policy at road segment $s_{i}$. Packet are forwarded $x_{i}$ times \\
$\eta$ & The solution to RIDE. \\
$H R N$ & The data collection latency constraint. \\
$L$ & Hierarchical road network generated from $R T M$. \\
\hline
\end{tabular}

provide (e.g., Google Map). The $v(s)$ and $d(s)$ can be detected by existing road infrastructures like inductive loops [18 or surveillance cameras [19]. If such infrastructure is temporarily not available, it is also possible to estimate these parameters using VANET [20].

We also assume the traffic conditions change slowly overtime. Here slowly means shorter than a data collection cycle (approximately 200 seconds). To justify this assumption, we extract some Cologne traffic trace data 21, which is also used in the evaluation. Fig. 3 shows a 30-minute trace and the corresponding statistic distribution when the traffic became congested gradually. The variation of average vehicle speed and number of vehicles per road segment is illustrated with different sample interval. We can see that within a data collection process, the traffic conditions can be regarded as static and the effect of variation can be ignored. This figure also illustrates that if we take macroscopic perspective (the entire traffic condition) rather than the microscopic one (the velocity of an individual vehicle), the results will not be influenced significantly.

The notations and symbols used in this paper are summarized in Table 1 .

\subsection{The RIDE Problem}

Time constraint is an important concern for many intelligent traffic related applications. Take the adaptive traffic light control application 22] for instance, traffic data must be collected to control the traffic light before vehicles pass it. Therefore, in this work, the time interval between data sensed from original vehicle and received by BS is designed as a hard constraint. And the total 

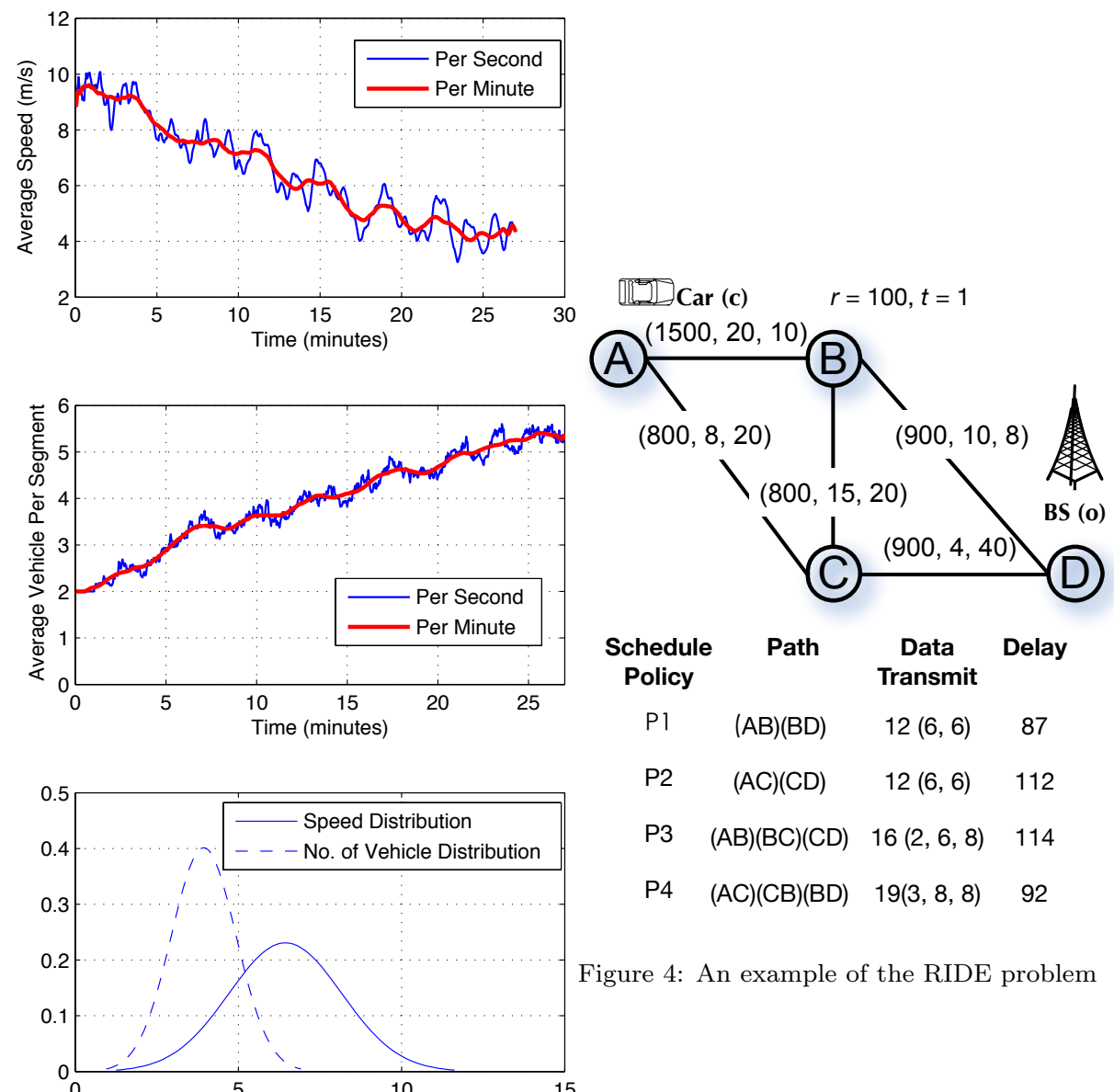

Figure 3: Variation of average vehicle speed and number of vehicles per road segment in traffic trace 
amount of data transmission via vehicular networks defines the network cost metric we want to minimize. In real world, the network cost can be wireless spectrum, bandwidth, and etc.

Fig. 4 illustrates an example of the problem. An $R T M$ with 5 road segments is given at the top. The values on the edges are the 3 -tuple properties of the corresponding road segments (length, speed and number of vehicles). The parameters for VANETs are mean transmission range $r=100$ and mean transmission latency $t=1$. Assume BS at intersection $D$ wants to collect data from a vehicle located at intersection $A$. There are multiple paths to forward the data along with 23. Moreover, data can either be transmitted via network or be carried on vehicles driving toward $D$. Therefore, there exists many possible ways to send the data. We name each of the possible solution as a scheduling policy.

Definition 2. (Scheduling Policy) A scheduling policy is a collection of consecutive road segments that data are forwarded along and the corresponding data forwarding count on each road segment. It is denoted as $\mathcal{P}=\left\{\left(s_{1}, x_{1}\right), \ldots,\left(s_{i}, x_{i}\right), \ldots,\left(s_{n}, x_{n}\right)\right\}$, where $s_{i}$ means data are forwarded along road segment $s_{i}$, and $x_{i}$ is data forwarding count, which mean on road segment $s_{i}$, data should be forwarded $x_{i}$ times.

The bottom of Fig. 4 lists some possible schedule policies $\mathcal{P}_{1}, \ldots, \mathcal{P}_{4} \cdot \mathcal{P}_{1}=$ $\left\{\left(s_{A B}, 6\right),\left(s_{B D}, 6\right)\right\}$ indicates data are transmitted along the path A-B-D and are forwarded 6 times at both segment $s_{A B}$ and $s_{B D}$. Therefore, the total data forwarding count is 12 and the total delay can be calculated as $6 \times 1+(1500-$ $6 \times 100) / 20+6 \times 1+(900-6 \times 100) / 10=87$. The delay of the rest schedule policies can also be calculated in the same way. If the application demand the data be collected within latency 100 , only $\mathcal{P}_{1}$ and $\mathcal{P}_{4}$ can satisfy the constraint, and data forwarding count for $\mathcal{P}_{1}$ is less than that of $\mathcal{P}_{4}$. However, we can observe that $\mathcal{P}_{1}$ is still not optimal, since reducing the data forwarding count to $11(5,6)$ can also satisfy the constraint (with delay $=91$ ).

With above preliminaries, the RIDE problem can be formally formulated as:

Definition 3. (The RIDE Problem)

Given:

1. Real-time Traffic Map $R T M=(N, E)$.

2. Base station located at $o \in N$. A vehicle located at $c \in N$ satisfies the data collection criterion.

3. Vehicular network with one hop average transmission range $r$ and transmission latency $t$.

4. Data collection latency $\eta$.

Objective:

Find a scheduling policy $\mathcal{P}$ to minimize:

$$
F=\sum_{\left(s_{i}, x_{i}\right) \in \mathcal{P}} x_{i}
$$




\section{Subject To:}

1.

$$
\sum_{\left(s_{i}, x_{i}\right) \in \mathcal{P}}\left(x_{i} \times t+\frac{l\left(s_{i}\right)-x_{i} \times r}{v\left(s_{i}\right)}\right) \leq \eta
$$

2.

$$
x_{i} \leq \min \left\{d\left(s_{i}\right)-1, \frac{l\left(s_{i}\right)}{r}\right\}
$$

In the problem formulation, $\eta$ is the latency constraint for data collection. The first part of Constraint 1) denotes the latency caused by data forwarding while the second part is the time spent on data carrying. Constraint 2) indicates data forwarding count is upper bounded by the number of vehicles on road (sparse traffic) and the division of road length and transmission range (dense traffic).

\subsection{Problem Hardness}

Now we prove the hardness of the problem. Specifically, we first prove the decision version of RIDE problem is NP-complete. The decision version of RIDE can be stated as: Does there exist a scheduling policy $\mathcal{P}$, such that the total data forwarding count $\sum_{\left(s_{i}, x_{i}\right) \in \mathcal{P}} x_{i}=k$, while the constraints are satisfied.

Theorem 1. The decision version of RIDE is NP-complete.

Proof We firstly prove the decision version of RIDE $\in$ NP, then prove RIDE $\in$ NP-hard.

To show RIDE $\in$ NP, suppose a schedule policy $\mathcal{P}$ is given. Clearly, we can verify the correctness of $\mathcal{P}$ in polynomial time. Specifically, the complexity of the verification algorithm is $O(n)$, where $n$ is the number of road segments in $\mathcal{P}$.

To show RIDE $\in$ NP-hard, we reduce the subset sum problem to it in polynomial time, as subset sum problem is NP-complete [24]. The subset sum problem can be formulated as : Given $n$ positive integers $\left\{a_{1}, \ldots, a_{n}\right\}$ and a target number $k$, find a subset $S \subseteq\left\{a_{1}, \ldots, a_{n}\right\}$ such that $\sum_{a_{i} \in S} a_{i}=k$. We present a polynomial time reduction that takes a set with $n$ integers and construct an instance of RIDE as follows:

Firstly, we construct the real-time traffic map $R T M$ in RIDE. The vertex set $N$ contains $2 \times n+1$ vertices. We create two vertices $i$ and $i^{\prime}$ for each integer $\left\{a_{1}, \ldots, a_{n}\right\}$ in subset sum, and a vertex $o$ as the base station. The road segment set $E$ contains $3 \times n$ road segments. We add one edge between $\left(a_{i}, a_{i}^{\prime}\right),\left(a_{i}, a_{i+1}\right)$ and $\left(a_{i}^{\prime}, a_{i+1}\right)$. Notice the exception that $a_{n}$ and $a_{n}^{\prime}$ should be connected to $o$. For each of the road segment in $E$, the 3 -tuple $\{l, v, d\}$ is constructed as $v=1$. For the length $l$, we set

$$
l(s)= \begin{cases}10 \times a_{i} & e(s)=a_{i}^{\prime} \\ 1, & \text { Otherwise }\end{cases}
$$




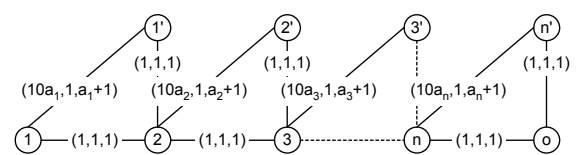

(a) Real-time Traffic Map Construction

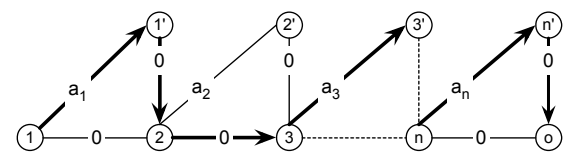

(b) Scheduling Policy Construction $\mathrm{S}=\left\{\mathrm{a}_{1}, \mathrm{a}_{3}, \ldots, \mathrm{a}_{\mathrm{n}}\right\}$

Figure 5: The reduction from subset sum to RIDE

For vehicle number $d$, we set

$$
d(s)= \begin{cases}a_{i}+1 & e(s)=a_{i}^{\prime} \\ 1, & \text { Otherwise }\end{cases}
$$

Fig. 5(a) shows the construction of $R T M$.

Secondly, we construct the vehicular network with $r=10$ and $t=1$. And the vehicle whose data shall be collected is located at vertex 1 . We then set the time constraint $\eta=n+k$. Finally, we construct the decision variable in decision version of RIDE $k^{\prime}$. We let $k^{\prime}=k$ in subset sum.

The above steps create an instance of decision version RIDE with all necessary conditions. We claim that the sum of subset $S \subseteq\left\{a_{1}, \ldots, a_{n}\right\}$, or $\sum_{a_{i} \in S} a_{i}=$ $k$, iff. there is a scheduling policy $\mathcal{P}$ such that the total data sent $\sum_{\left(s_{i}, x_{i}\right) \in \mathcal{P}} x_{i}=$ $k^{\prime}$.

$(\Rightarrow)$ Assume $\left\{a_{1}, \ldots, a_{n}\right\}$ contains a subset $S$ that satisfy $\sum_{a_{i} \in S} a_{i}=k$. In the constructed RIDE instance, the schedule policy $\mathcal{P}$ can be obtained as:

$\forall\left(s_{i}, x_{i}\right) \in \mathcal{P}$,

$$
\begin{gathered}
s_{i}= \begin{cases}\left(i, i^{\prime}\right) \cup\left(i^{\prime}, i+1\right), & a_{i} \in S \\
(i, i+1), & \text { Otherwise }\end{cases} \\
x_{i}= \begin{cases}a_{i}, & s_{i}=\left(i, i^{\prime}\right) \\
0, & \text { Otherwise }\end{cases}
\end{gathered}
$$

Fig. 5 (b) shows an example of the solution. It is easy to verify that $\sum_{\left(s_{i}, x_{i}\right) \in \mathcal{P}} x_{i}=$ $k^{\prime}$. In addition, the two constraints can also be satisfied. For constraint 1), $\eta=n+k$ always hold. For constraint 2), $\min \left\{d\left(s_{i}\right)-1, \frac{l\left(s_{i}\right)}{r}\right\}=a_{i}$.

$(\Leftarrow)$ If the constructed instance of RIDE exists a policy $\mathcal{P}$ that satisfies the decision criterion, the solution to the subset sum is equivalent to the union of $\left\{x_{i}\right\}, \forall\left(s_{i}, x_{i}\right) \in \mathcal{P}$.

Since RIDE belongs to the class NP and is NP-hard, we can conclude that it is NP-complete. 


\subsection{ILP Formulation of RIDE}

The RIDE problem can also be mathematically formulated as an integer linear programming (ILP) problem. The ILP formulation can help to better understand the problem. Moreover, with ILP formulation, the problem can be directly passed to the existing ILP solvers to obtain the optimal solutions. However, formulating the problem as ILP does not reduce the computational complexity, as ILP itself is also NP-complete [24.

First, the real-time traffic map can be re-written as three matrices $l_{i j}, v_{i j}, d_{i j}$ representing each element in the 3 -tuple of road segment, respectively. If there is no road segment between vertex $i$ and $j$, we set $l_{i j}=\infty, v_{i j} \rightarrow+0, d_{i j}=1$.

Then, we define variables $x_{i j}$ as:

$$
x_{i j}= \begin{cases}0, & \text { Data are not forwarded along path } i j \\ \geq 0, & \text { Otherwise }\end{cases}
$$

Then, $x_{i j}-1$ is the data forwarding count on path $i j$. In this way, the RIDE problem can be formulated in ILP form as follows:

Minimize:

$$
\sum_{i=1}^{i=n} \sum_{j=1}^{j=n} x_{i j}
$$

Subject To:

$$
\begin{aligned}
& \sum_{i=1}^{i=n} \sum_{j=1}^{j=n}\left(t-\frac{r}{v_{i j}}\right) x_{i j} \leq \eta-\sum_{i=1}^{i=n} \sum_{j=1}^{j=n} \frac{l_{i j}}{v_{i j}} \\
& x_{i j} \leq d_{i j}-1 \\
& x_{i j} \leq \frac{l_{i j}}{r} \\
& \sum_{j} \frac{x_{i j}}{x_{i j}}-\sum_{j} \frac{x_{j i}}{x_{j i}}= \begin{cases}1 & i=c \\
-1 & i=o \\
0 & \text { Othrwise }\end{cases} \\
& x_{i j} \in \mathbb{N}^{0}
\end{aligned}
$$

Equation 8 is transformed from the original Constraint 1). Equation 9 and Equation 10 are transformed from the original Constraint 2). Equation 11 ensures there is only one path from source $c$ to destination $o$. Equation 12 ensures that the solution must be integers.

Since ILP remains NP-complete. It is still time-consuming to directly utilize existing ILP solvers. Therefore, we still have to find ways to reduce the computational complexity. 


\section{Solution To RIDE}

Since RIDE belongs to NP-complete, there is no polynomial time solution unless $\mathrm{P}=\mathrm{NP}$. Although we have formulated the problem in ILP form, and use ILP solver to find the solution. The ILP solvers are quite general and not dedicatedly optimized for the RIDE problem. To solve the RIDE problem in better ways, we propose two approaches for different scenarios, one uses dynamic programming(DP) and the other uses genetic algorithm(GA).

\subsection{Road Topology Hierarchicalization}

Before introducing the details of the solution, we want to introduce a simple and neat road topology to make the problem easier to be solved and understood. In reality, the topology of road networks can be potentially complicated. We would like to pre-process the road network to make it comprehensible. The basic idea is to label the road segments using different layers according to the distance to BS and then transform the road network into a hierarchical graph.

Definition 4. (Hierarchical Road Network) Hierarchical Road Network can be defined as a k-partite graph $H R N=(N, E)$. Where $E$ is the set of all road segments and $N$ is the set of all road segments intersections. A road segment can be defined as the same 3-tuple as in Road Networks. Each intersection is also defined by 3-tuple $\{x, y, L\}$, where $x, y$ are the position of the intersection. $L$ is the layer label and $L \in\{L 0, L 1, \ldots, L n\}$. The $B S$ is labeled as $L 0$ or layer 0 . The graph is hierarchical iff $\forall s \in E,|b(s) . L-e(s) . L|=1$.

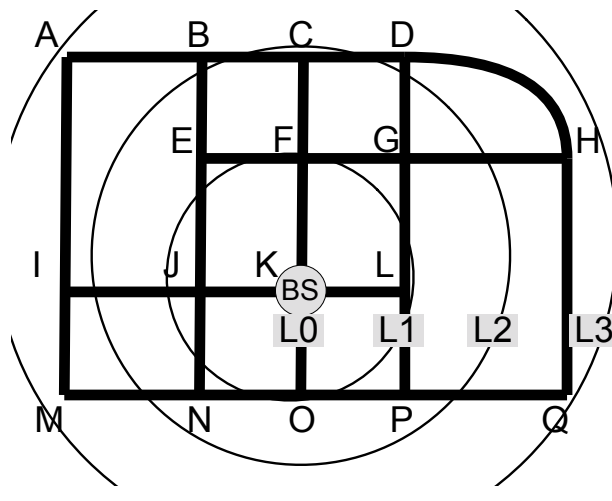

Figure 6: A sample road topology

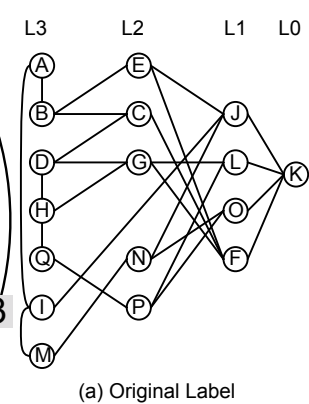

Figure 7: Hierarchicalization of the sample road topology

Fig. 6illustrates an example of road topology. Fig. 7 shows how to label and transform the sample road topology to hierarchical road network. The algorithm is listed in Algorithm 1. The complexity of Algorithm 1 is $O(m \times n)$, where $m$ is the number of edge and $n$ is the number of vertex. The algorithm only need to run once for each road network since it is deterministic. Notice that when adjusting road segments, the property $l(s)$ and $d(s)$ should also be adjusted accordingly. The adjustment is not presented in Algorithm 1 for simplicity. 


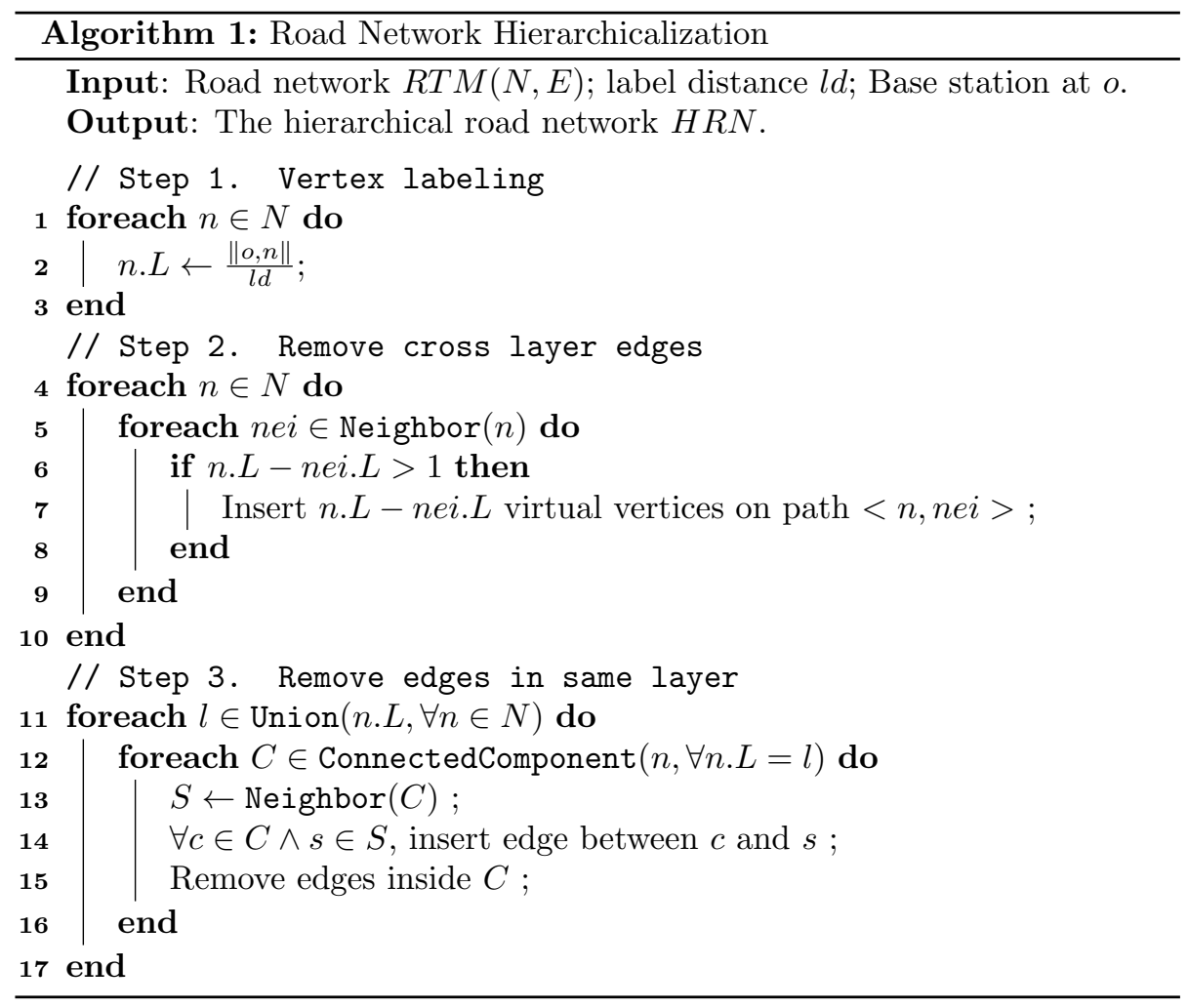

\subsection{Dynamic Programming based Solution}

Some applications may only require small-scaled data collection. The number of vehicles involved may not be large (e.g., less than 100). In this case, we can use dynamic programming to find the optimal solution. To solve the problem using dynamic programming, a recursive definition of the problem must be found. We write the recursive function for RIDE as:

$$
\begin{aligned}
& F(s, d, t)= \\
& \begin{cases}\operatorname{mfc}(s, d, t), & \text { s.L }-d . L=1 \\
\min _{\left\{t_{i} \leq t, s_{i} \in S_{n}\right\}}\left\{F\left(s_{i}, d, t_{i}\right)+F\left(s, s_{i}, t-t_{i}\right)\right\}, & \text { Otherwise }\end{cases}
\end{aligned}
$$

In Equation $13, s$ and $d$ are the source and destination intersections, respectively. $t$ is the remaining time before data collection constraint $\eta$ expires. $S_{n}$ means the possible next road segment candidates for intersection $s . \operatorname{mfc}(s, d, t)$ is the function to calculate the minimum data forwarding count for single road segment from $s$ to $d$ in given time constraint $t$. The first case in Equation 13 shows if the source and destination is on two ends of the same road segment, we 


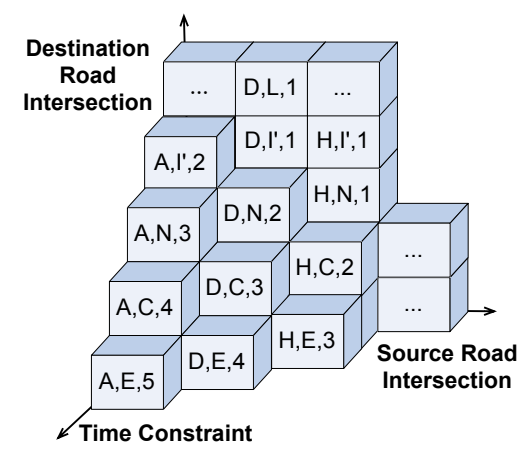

Figure 8: Sub-problem division in dynamic programming: the solutions of the sub-problems are stored in the $3 \mathrm{D}$ table to avoid duplicated calculation

can simply return the minimum data forwarding count for the segment. Otherwise, we enumerate the combination of all remaining time and candidate road segments, and choose the minimum results.

The function $\mathrm{mfc}(s, d, t)$ is defined as:

$$
m f c(s, d, t)=
$$

$$
\begin{cases}0, & t \times v\left(s_{s d}\right) \geq l \\ +\infty & t<d\left(s_{s d}\right) \times t+\frac{l\left(s_{s d}\right)-d\left(s_{s d}\right) \times r}{v\left(s_{s d}\right)} \\ \left\lceil\frac{t \times v\left(s_{s d}\right)-l\left(s_{s d}\right)}{t s \times v\left(s_{s d}\right)-r}\right\rceil, & \text { Otherwise }\end{cases}
$$

The first case indicates the situation that carrying only can satisfy the constraint, thus no data forwarding is required. The second case shows even data forwarding with maximum effort can not satisfy the time constraint, so there is no solution. The third case shows the minimum data forwarding to satisfy the constraint. Notice the equation may not be integer, so we use the ceiling value.

The basic idea of dynamic programming is to divide the problem into several sub-problems that could be duplicated. In our case, according to the recursive definition, the problem is divided into sub-problems with 3 -tuple $(s, d, t)$ as keys to differentiate with each others, where $s$ is source road intersection, $d$ is destination road intersection and $t$ is remaining time constraint. Fig. 8 illustrates the $3 \mathrm{D}$ table for storing the solution of divided sub-problems.

The complete dynamic programming algorithm is listed in Algorithm 2 Dynamic programming is a typical substitution of space for time solution, therefore the space complexity is an important concern. In our solution, the space complexity of the algorithm is upper bounded by $O\left(n^{2} \times \eta\right)$, where $n$ is the number of road intersections and $\eta$ is the time constraint. The output of Algorithm 2 is only the minimum value of data forwarding count. To get the scheduling policy $\mathcal{P}$, a backtrace in $G T$ is necessary. 


\subsection{Genetic Algorithm based Solution}

For some other data collection applications, the road segments involved may be extremely large, the previous dynamic programming based solution may not be efficient enough since the worst case complexity of DP is still exponential. In this case, we should consider some heuristic optimization methods and bear with the near optimal solution. In this section, we propose a genetic algorithm (GA) based solution for this scenario.

To develop a GA algorithm, several issues should be addressed. They are: genetic representation, population initialization, selection, genetic operators (crossover, mutation) and termination. We will introduce the detailed implementation one by one.

\subsubsection{Genetic representation and population initialization}

Genetic representation answers how to encode a solution with a chromosome or a string. In this work, with the help of the hierarchical road networks, the solution can be represented by a fixed length chromosome. Therefore, compared with other GA based routing solutions with variable length chromosome [25], our approach is more simple and efficient.

In our solution, the chromosome length is fixed to $2 \times \max (v \cdot L), \forall v \in H R N$. Two loci are used to encode each $p_{i}=\left(r s_{i}, x_{i}\right)$ in $\mathcal{P}$. Fig. 9 depicts an example for the representation. In the figure, data are forwarded along trajectory $\{\mathrm{Q}$, $\mathrm{P}, \mathrm{O}, \mathrm{K}$, the data forwarding counts are labeled on the edge. That is, data are forwarded 6 times on $(\mathrm{Q}, \mathrm{P}), 5$ times on $(\mathrm{P}, \mathrm{O})$ and 3 times on $(\mathrm{O}, \mathrm{K})$. Therefore, the solution can be encoded as 'Q6P5O3K0'. Notice that the last 0 in the string is only for padding and has no physical meaning.

At the beginning of the algorithm, we initialize the first generation of population randomly. The generation algorithm works as follows: Firstly, it chooses a random neighbor of the source intersections in next layer, then it assigns a data forwarding count randomly according to Constraint 2$)$ in the range $[0$, $\left.\min \left\{d\left(s_{i}\right)-1, \frac{l\left(s_{i}\right)}{r}\right\}\right]$. After that, it continues for the next neighbor in next layer. The iteration continues until the base station is reached.

\subsubsection{Selection}

After the first generation of population is generated, we will use fitness function to evaluates the quality of these generated solutions. In RIDE, the fitness function is obvious because our objective is to minimize the data transmission overhead. Therefore, the fitness function is the summation of the integers at even locus. In Fig. 9, the result of fitness function is $14=6+5+3+0$.

For each of the generations, the algorithm will select certain amount of the populations as survivals according to the fitness function. These survivals are used to produce next generations via genetic operators.

\subsubsection{Genetic operators}

In this work, we consider three kinds of genetic operators: crossover, mutation and repair. 


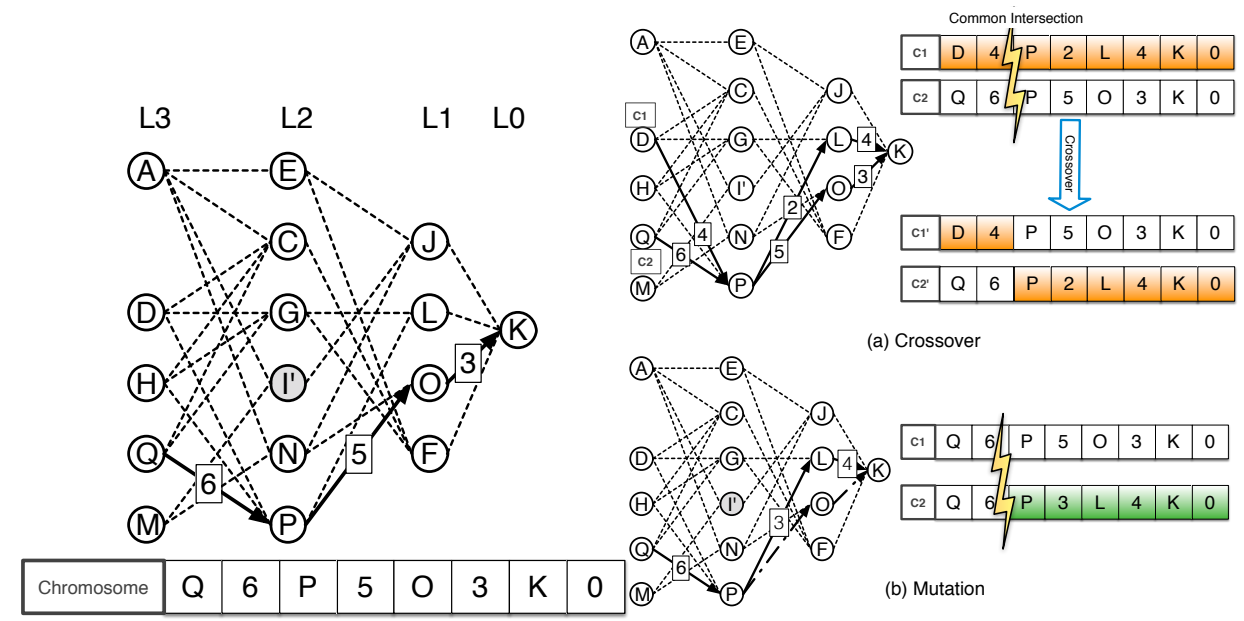

Figure 9: Chromosome representation of the Figure 10: (a) Crossover of two chromosomes: solution

Exchange the content of the two solutions at right side of common intersection $P$. (b) $\mathrm{Mu}-$ tation of one chromosome: Change the data forwarding policy after intersection $P$.

Crossover operation takes two individual chromosomes from survived population and generates new individuals by combination. In this work, the crossover is implemented as follows: we firstly find common intersections except source and destination of two given chromosomes. Then choose one of them randomly as cross point and swap the contents of the two inputs. If there are no common intersections, no operation is done. Fig. 10(a) shows an example of crossover.

Mutation operation simulates the same phenomenon in natural world. With certain probability, the content of a chromosome is accidentally changed. In GA, this operation can help to prevent returning local optimal solutions. However, in this work, changing a single road intersection or segment may not ensure the new solution is still a valid trajectory. Therefore, we randomly choose a road intersection in the given solution and replace the remaining path on the right with new ones. Fig. 10(b) shows an example of mutation.

In many cases, the initialization, crossover and mutation operation can not generate a valid solution. E.g., the time threshold can not be satisfied. If the ratio of invalid solutions become large, the performance of the algorithm will be reduced. Therefore, we should repair those invalid solutions. In this work, after each new individual is generated, we will firstly check if it is a valid solution by verifying Constraint 1). We needn't check Constraint 2) since it has been considered at initialization phase. If Constraint 1) is violated, we keep increasing the data forwarding count on the road segment with the lowest vehicle speed until it is satisfied. If we still can not repair the solution by adding the data forwarding count on all segments to maximum possible values, the solution is discarded. 


\subsubsection{Termination}

In our solution, the iterations of creating new generations are terminated if one of the following conditions is satisfied: 1) An optimal solution $F=0$ is found, which means no data forwarding is required. 2) The predefined number of iterations has been reached.

\section{Data Collection Protocol Design}

The network protocol can be divided into two phases: request dissemination phase and data collection phase. The former is used by BS to deliver the data collection request to nearby vehicles and the latter is used to collect the requested data back to BS.

In request dissemination phase, the requests are all initiated by the base station and then disseminated to all vehicles in the given ROI. In our solution, we use epidemic style dissemination [26 to complete the task because it is simple, efficient and reliable. Since the size of request dissemination packet is limited, the overhead is also tolerable.

In our implementation, the solution to RIDE is computed distributedly at each vehicle on demand. Therefore, vehicle need to know the real-time traffic information. To tell vehicles the information, we send them along with the data collection request sent by $\mathrm{BS}$ each time, the $H R N$ can be compressed to $2 \mathrm{~KB}$, the overhead is acceptable. After vehicles receive the request and traffic information, the schedule policy $\mathcal{P}$ can be computed on vehicle side. And then, the solution can instruct the vehicles on how to send data back to the BS.

In data collection phase, data are forwarded according to the scheduling policy $\mathcal{P}$. The flowchart of the data collection protocol is illustrated in Fig. 11. When data arrive at a new road segment, vehicle will forward the data to furthest vehicle as long as the forwarding count has not exceeded the value in the schedule policy. Then, vehicle will carry the data until next road intersection, where the data can be forwarded to vehicles running at next road segment. It is possible that the forwarding could fail due to low traffic density or network failure. Worse more, vehicle carrying the data may heading to a different road segment due to the uncontrollable mobility nature of VANETs. In this case, schedule policy $\mathcal{P}$ should be recalculated according to current position. The algorithm will not stop until data arrive at BS.

\section{Performance Evaluation}

\subsection{Evaluation Design and Experiment Setup}

To evaluate the performance of RIDE, the most important metric is the improvement of using RIDE in vehicular data collection applications, especially the comparison with some existing data collection protocols. In addition, the time complexity and the approximation ratio of different solutions are also need to be evaluated. 


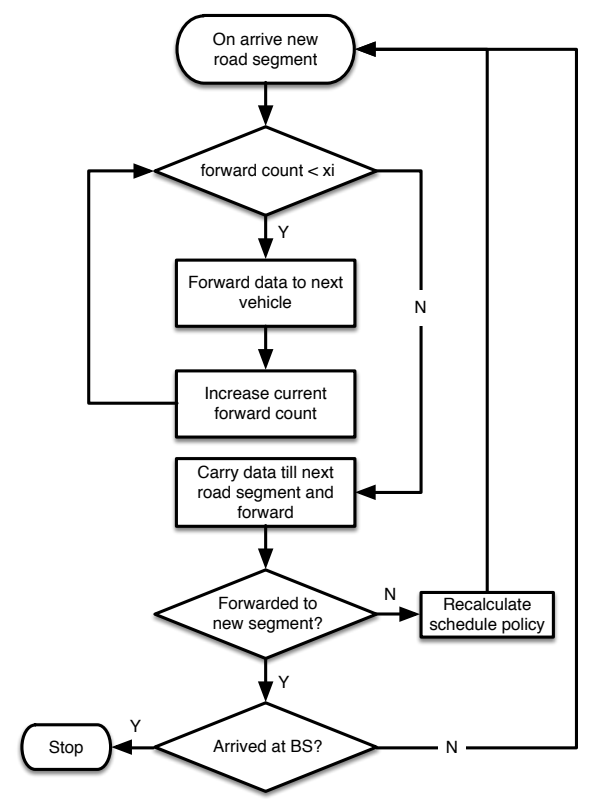

Figure 11: Flowchart for data collection protocol

To make the evaluation realistic and convincing, we use traffic trace data set of Cologne, Germany from TAPASCologne project 21. Fig. 12 shows the work-flow of our experiment and evaluation. Firstly, the data set is imported to SUMO traffic simulator 27] to generate real-time traffic trace, then the output is pre-processed by Python scripts to generate real-time traffic map and hierarchical road networks required as the input of the algorithm. Then, we apply the dynamic programming and genetic algorithm solutions to it. In this way, the complexity of different solutions can be obtained. Finally, we use network simulator NS-3 28 to run the data collection application according to the scheduling policy. Then, the evaluation results for different data collection protocols are obtained. The evaluation is run on a virtual machine with $1.6 \mathrm{GHz}$ CPU, 512MB RAM and Ubuntu OS.

\subsection{RIDE in Data Collection Applications}

To evaluate the performance of RIDE in data collection applications, we implemented the network protocol in NS-3. The solution is implemented as an application layer protocol on top of the UDP protocol. Other configuration parameters can be found in Table 2 .

We compare RIDE with DB-VDG [12] and epidemic style data collection 26]. DB-VDG have similar scenarios and objective with RIDE but do not consider real-time traffic information. Specifically, we compare with DB-VDG SBSS since the effectiveness is higher than DB-VDG DBSS. Epidemic solution 


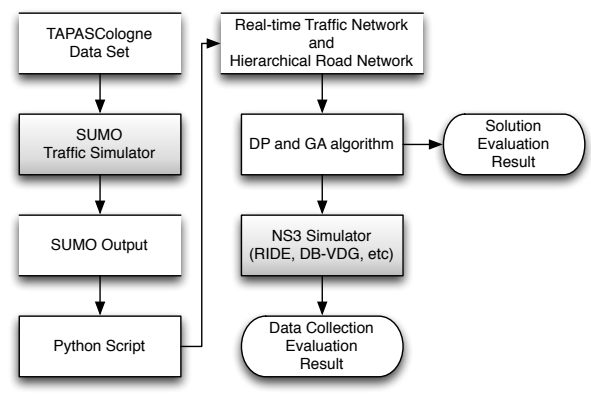

Figure 12: Flowchart for experiment and evaluation

means we use the same best-effort protocol for query dissemination and data collection.

We are interested in two metrics: effectiveness and efficiency. They are defined as follows:

$$
\begin{gathered}
\text { Effectiveness }=\frac{N_{\text {delivered }}}{N_{\text {vehicles }}} \% \\
\text { Efficiency }=\frac{D_{\text {delivered }}}{D_{\text {vehicles }}} \%,
\end{gathered}
$$

where $N_{\text {delivered }}$ is the number of vehicles whose data is delivered to the BS before time constraint expiration and $N_{\text {vehicles }}$ is the number of vehicles whose data should be delivered to the BS. $D_{\text {delivered }}$ is the amount of data received by the BS and $D_{\text {vehicles }}$ is the total amount of data sent by involved vehicles. The main reason we choose these two metrics is that different applications may have different requirements for data collection. E.g., some time-critical applications require that data should be delivery at all costs. In this case, effectiveness is a better metric. On the contrary, those cost sensitive applications may prefer to use efficiency as the major metric.

We observe the effectiveness and efficiency of the three approaches with different traffic density. We only focus on the change of traffic density mainly because of the fact that the traffic speed, traffic flow and traffic density have some fundamental correlations [29]. It is possible to use one result to infer the other two.

Fig. 13(a) and (b) show the effectiveness and the efficiency of the three solutions, respectively. The effectiveness of epidemic solution outperforms others in all circumstance since it always tries to forward data whenever possible. Therefore, the efficiency of epidemic solution is the worst since many unnecessary forwarding are carried out. There is no significant difference between RIDE and DB-VDG in terms of efficiency. However, RIDE outperforms DB-VDG in effectiveness especially when the traffic density is low. This is because DB-VDG tries to carry as long as possible to reduce the network overhead. But when traffic density is low, it is very likely that when DB-VDG decide to forward data, there 
(a) Effectiveness

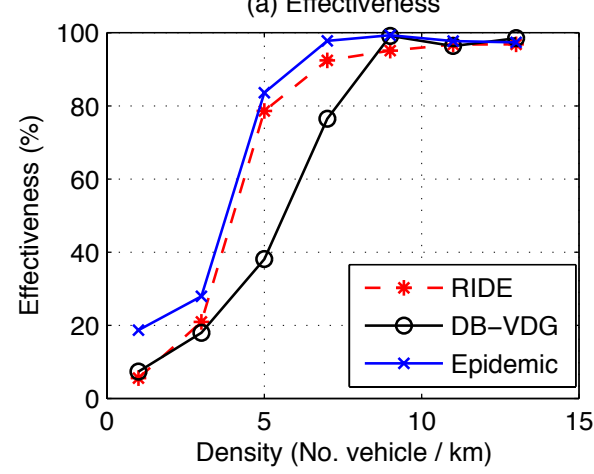

(b) Efficiency

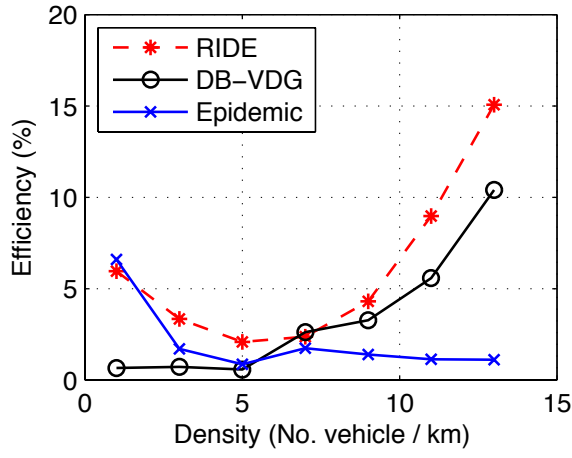

Figure 13: Evaluation of RIDE in data collection applications (a) Number of Vehicles

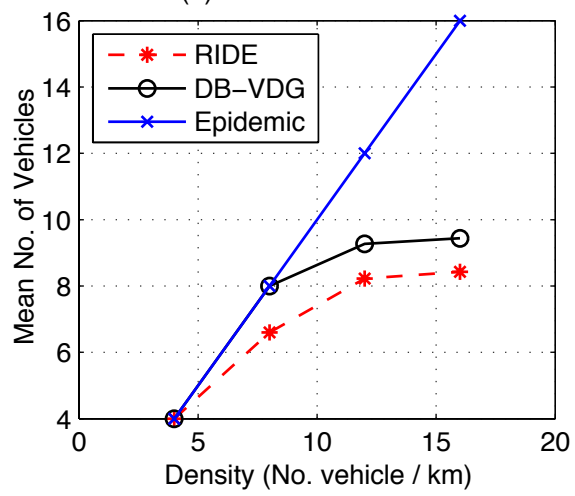

(b) Number of Hops

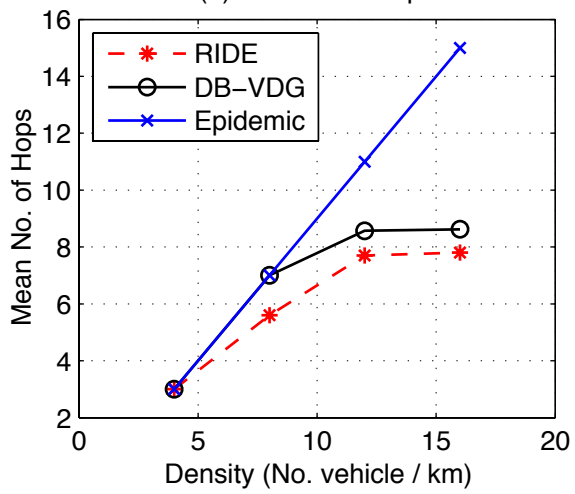

Figure 14: Number of Vehicles/Hops involved in data collection applications

is no next hop candidate nearby and this is the major cause of deadline missing in DB-VDG. RIDE can overcome this issue by scheduling according to traffic information in advance. In dense traffic scenario, the effectiveness of RIDE and DB-VDG is close since DB-VDG can always find next hop candidate.

Fig. 14(a) and (b) illustrate the mean number of vehicles and the number of hops involved in the experiment, with respect to the change of traffic density. From these figures, we can find out that different protocol has different characteristics. Specifically, in the epidemic style protocol, all vehicles are involved and the maximum number of hops is reached. Differently, our proposed protocol can reduce the number of hops required to reach the BS, due to the dynamic scheduling we apply.

In summary, in urban traffic collection applications, DB-VDG is suitable for dense traffic scenario. If real-time traffic information can be obtained, RIDE works fine under both dense and sparse traffic condition. If the effectiveness is the most important concern, the data packet size is not large and the number 
(a)
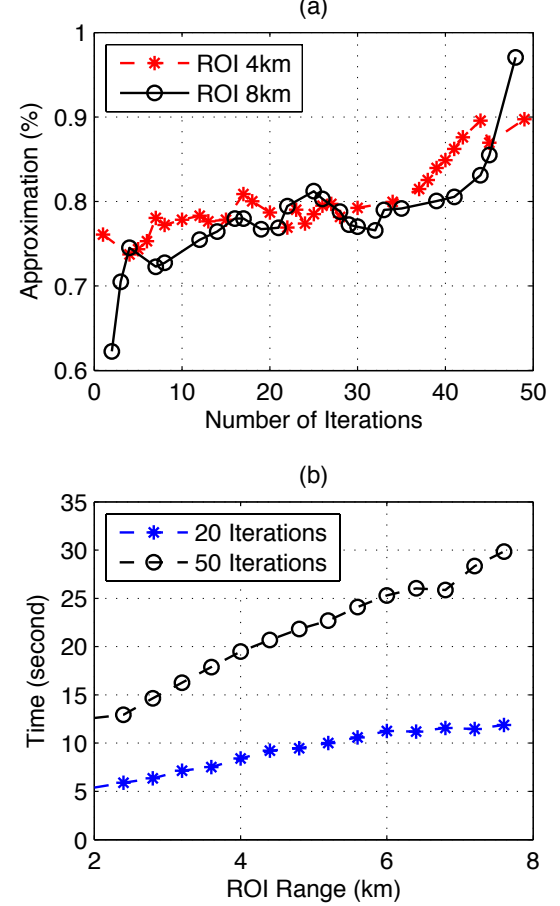

Figure 15: Evaluation of genetic algorithm 


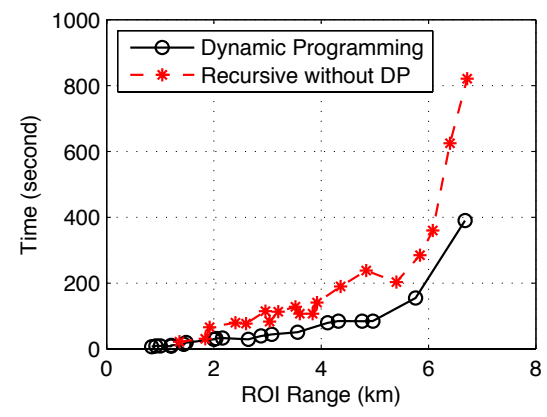

Figure 16: Time complexity of dynamic programming

of vehicles satisfying collection query is not large, epidemic solution can also be considered.

\subsection{Computational Complexity and Accuracy}

As we have analyzed, dynamic programming can always get the optimal results. However, the complexity is unaffordable when the input gets large. Therefore, we want to evaluate the computational complexity for dynamic programming based solution. The time spend on calculating the results increases with the size of ROI.

Fig. 16 illustrates the time complexity of dynamic programming. We compare DP with recursive only solution (Remove line 3-5 in Algorithm 2). We can see that DP can reduce the computation time by $2 / 3$. However, with the increase of input, the time still increases exponentially. Therefore, DP is only suitable for small-scaled data collection scenario.

Compared with DP, the advantage of the genetic algorithm is that the execution time is controllable by adjusting the maximum iteration, population number and other parameters. However, the results of GA are sub-optimal. Therefore, we are interested in the relationship among time cost, approximation and topology complexity. In the experiment, we fix the population size to be 10000 .

Fig. 15 shows the evaluation results for GA. Fig. 15(a) shows the approximation ratio of the algorithm. From the results, we can see that the approximation ratio has a steady increase trend with the iteration number. However, there are uncertainty in the results since GA has no theoretical bound. Fig. 15(b) shows the time cost as the chromosome length increases. The time cost increases linearly. Since the chromosome length is determined by the maximum layer number in $H R N$ and the layer number determines the scale of the data collection scenario. Therefore, GA can be applied for large scaled data collection scenario. 


\section{Discussion}

\subsection{Data aggregation}

In current implementation of RIDE, the data originating from different sources is not aggregated together before sending to the BS. We notice that doing data aggregation [30] in the network whenever possible would help to reduce the amount of data to be transmitted. Actually, data aggregation, especially opportunistic data aggregation and our data forwarding scheduling is not conflict. Moreover, they are complementary and can be used simultaneously in data collection. Our preliminary work has shown that if a data aggregation function can be found for specific data type, the total amount of data to be transmitted can be significantly reduced. E.g., If the BS wants to know the mean traffic speed around. It would be much better to calculate the mean speed of vehicle while transmitting, rather than collecting the speed of each individual vehicle and calculate at the base station.

\subsection{Data Integrity}

In the real world applications, the vehicular networks usually include several malicious nodes, which will not collaborate with other nodes, or even attempt to destroy the data integrity on purpose. This paper assumes that there is no malicious node that participate the data collection process. Therefore, to make this work practical, additional data integrity protocols should be applied together.

\subsection{Computational complexity}

Since the scheduling policy is computed at each vehicle on demand, and Fig. 16 and Fig. 15 show that the computation complexity is considerably high. Therefore, the approach has potential practical issue. In fact, first today's mainstream vehicle onboard computer is much powerful than the virtual machine we use (1.6GHz CPU, 512MB RAM). In addition, vehicles can also utilize mobile cloud partitioning 31 to speed up the computation.

\section{Conclusion}

We addressed the problem of a BS collecting data from surrounding vehicles. Taking into account the real-time traffic information, a data collection solution RIDE are designed to minimize the communication cost. We proved the problem to be NP-complete and gave two solutions for different scenarios. Evaluations

showed that RIDE outperforms some existing solutions in terms of efficiency and effectiveness. 


\section{Acknowledgment}

This work is partially supported by the National Science Foundation of China (Grant No.61472283 and 61103185), the Fok Ying-Tong Education Foundation, China (Grant No. 142006), the Fundamental Research Funds for the Central Universities (Grant No. 2013KJ034 and No. 2100219043). This project is also sponsored by the Scientific Research Foundation for the Returned Overseas Chinese Scholars, State Education Ministry.

[1] T. Qiu, L. Chi, W. Guo, Y. Zhang, Stets: A novel energy-efficient time synchronization scheme based on embedded networking devices, Microprocessors and Microsystems 39 (8) (2015) 1285 - 1295.

[2] T. Qiu, D. Luo, F. Xia, N. Deonauth, W. Si, A. Tolba, A greedy model with small world for improving the robustness of heterogeneous internet of things, Computer Networks 101 (2016) 127 - 143.

[3] V. Bychkovsky, K. Chen, M. Goraczko, H. Hu, B. Hull, A. Miu, E. Shih, Y. Zhang, H. Balakrishnan, S. Madden, Data management in the cartel mobile sensor computing system, in: ACM SIGMOD international conference on Management of data (SIGMOD), 2006, pp. 730-732.

[4] M. Ylizaliturri-Salcedo, S. Delgadillo-Rodriguez, J. Garcia-Macias, M. Tentori, Participatory sensing for improving urban mobility, in: Ubiquitous Computing and Ambient Intelligence. Context-Awareness and ContextDriven Interaction, Vol. 8276 of Lecture Notes in Computer Science, Springer International Publishing, 2013, pp. 378-381.

[5] P. Papadimitratos, A. La Fortelle, K. Evenssen, R. Brignolo, S. Cosenza, Vehicular communication systems: Enabling technologies, applications, and future outlook on intelligent transportation, IEEE Communications Magazine 47 (11) (2009) 84-95.

[6] C.-H. Lo, W.-C. Peng, C.-W. Chen, T.-Y. Lin, C.-S. Lin, CarWeb: A traffic data collection platform, in: International Conference on Mobile Data Management (MDM), 2008, pp. 221-222.

[7] S.-C. Hu, Y.-C. Wang, C.-Y. Huang, Y.-C. Tseng, A vehicular wireless sensor network for CO2 monitoring, in: IEEE Sensors, 2009, pp. 14981501 .

[8] H. Zhu, M. Li, L. Fu, G. Xue, Y. Zhu, L. Ni, Impact of traffic influxes: Revealing exponential intercontact time in urban VANETs, IEEE Transactions on Parallel and Distributed Systems 22 (8) (2011) 1258-1266.

[9] S. Zhao, L. Fu, X. Wang, Q. Zhang, Fundamental relationship between nodedensity and delay in wireless ad hoc networks with unreliable links, in: International conference on Mobile computing and networking (MobiCom), 2011, pp. 337-348. 
[10] U. Lee, E. Magistretti, M. Gerla, P. Bellavista, A. Corradi, Dissemination and harvesting of urban data using vehicular sensing platforms, IEEE Transactions on Vehicular Technology 58 (2) (2009) 882-901.

[11] I. Salhi, M. Cherif, S. Senouci, A new architecture for data collection in vehicular networks, in: IEEE International Conference on Communications (ICC), 2009, pp. 1-6.

[12] C. E. Palazzi, F. Pezzoni, P. M. Ruiz, Delay-bounded data gathering in urban vehicular sensor networks, Pervasive and Mobile Computing.

[13] L. Guo, R. Beyah, Y. Li, SMITE: A stochastic compressive data collection protocol for mobile wireless sensor networks, in: IEEE INFOCOM, 2011, pp. 1611-1619.

[14] Y. Gu, D. Bozda, R. W. Brewer, E. Ekici, Data harvesting with mobile elements in wireless sensor networks, Computer Networks 50 (17) (2006) 3449-3465.

[15] J. Burgess, B. Gallagher, D. Jensen, B. N. Levine, MaxProp: Routing for vehicle-based disruption-tolerant networks, in: IEEE INFOCOM, 2006, pp. $1-11$.

[16] A. Balasubramanian, B. Levine, A. Venkataramani, Replication routing in DTNs: A resource allocation approach, IEEE/ACM Transactions on Networking 18 (2) (2010) 596-609.

[17] H.-Y. Huang, P.-E. Luo, M. Li, D. Li, X. Li, W. Shu, M.-Y. Wu, Performance evaluation of suvnet with real-time traffic data, IEEE Transactions on Vehicular Technology 56 (6) (2007) 3381-3396.

[18] S. Oh, S. Ritchie, C. Oh, Real-time traffic measurement from single loop inductive signatures, Transportation Research Record: Journal of the Transportation Research Board 1804 (2002) 98-106.

[19] T. Schoepflin, D. Dailey, Dynamic camera calibration of roadside traffic management cameras for vehicle speed estimation, IEEE Transactions on Intelligent Transportation Systems 4 (2) (2003) 90-98.

[20] Z. He, J. Cao, T. Li, MICE: A real-time traffic estimation based vehicular path planning solution using VANETs, in: International Conference on Connected Vehicles and Expo (ICCVE), 2012.

[21] S. Uppoor, M. Fiore, Large-scale urban vehicular mobility for networking research, in: IEEE Vehicular Networking Conference (VNC), 2011, pp. 6269.

[22] B. Zhou, J. Cao, H. Wu, Adaptive traffic light control of multiple intersections in WSN-based ITS, in: Vehicular Technology Conference (VTC), 2011, pp. 1-5. 
[23] T. Qiu, W. Sun, Y. Bai, Y. Zhou, An efficient multi-path self-organizing strategy in internet of things, Wireless Personal Communications 73 (4) (2013) 1613-1629.

[24] M. R. Garey, D. S. Johnson, Computers and Intractability; A Guide to the Theory of NP-Completeness, W. H. Freeman and Co., New York, NY, USA, 1990.

[25] C. W. Ahn, R. S. Ramakrishna, A genetic algorithm for shortest path routing problem and the sizing of populations, IEEE Transactions on Evolutionary Computation 6 (6) (2002) 566-579.

[26] M. Nekovee, Epidemic algorithms for reliable and efficient information dissemination in vehicular, IET Intelligent Transport Systems 3 (2) (2009) $104-110$.

[27] M. Behrisch, L. Bieker, J. Erdmann, D. Krajzewicz, Sumo - simulation of urban mobility: An overview, in: International Conference on Advances in System Simulation (SIMUL), Barcelona, Spain, 2011.

[28] G. F. Riley, T. R. Henderson, The NS-3 network simulator, in: Modeling and Tools for Network Simulation, Springer Berlin Heidelberg, 2010, pp. $15-34$.

[29] N. Geroliminis, C. F. Daganzo, Existence of urban-scale macroscopic fundamental diagrams: Some experimental findings, Transportation Research Part B: Methodological 42 (9) (2008) 759-770.

[30] S. Dietzel, F. Kargl, G. Heijenk, F. Schaub, Modeling in-network aggregation in VANETs, IEEE Communications Magazine 49 (11) (2011) 142-148.

[31] L. Yang, J. Cao, Y. Yuan, T. Li, A. Han, A. Chan, A framework for partitioning and execution of data stream applications in mobile cloud computing, ACM SIGMETRICS Performance Evaluation Review 40 (4) (2013) $23-32$. 


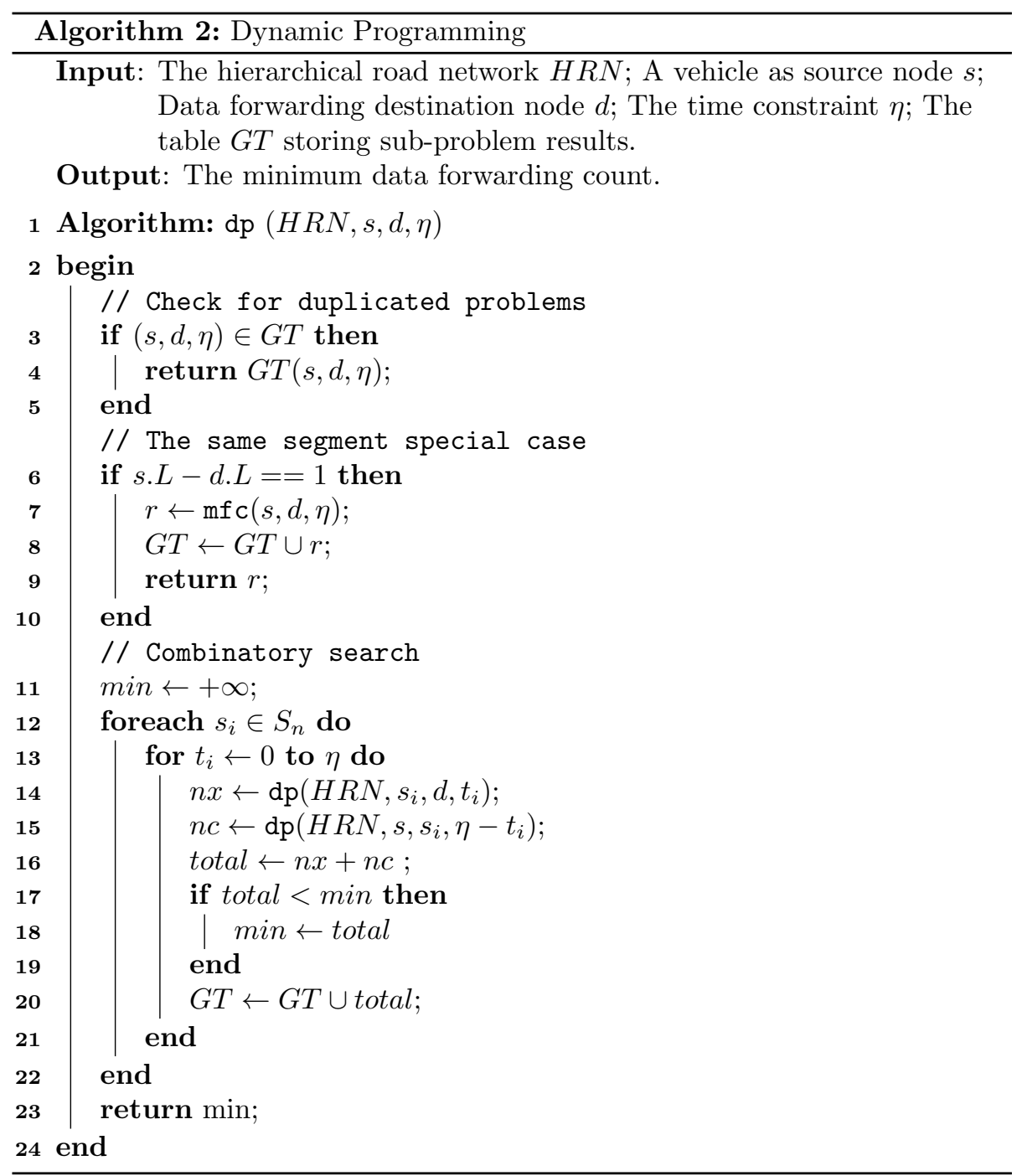

Table 2: NS-3 Simulation Parameters

\begin{tabular}{c|c}
\hline Parameter & Value \\
\hline MAC/PHY Standard & IEEE 802.11p SCH \\
Propagation delay mode & Constant Speed \\
MAC type & Adhoc Wif Mac \\
Propagation loss model & Range Propagation Loss Model \\
Transmission range & $200 \mathrm{~m}$ \\
Simulation period & 300 seconds \\
\hline
\end{tabular}

